\title{
CONSISTENT LEAST SQUARES ESTIMATOR FOR CO-ARRAY-BASED DOA ESTIMATION
}

\author{
Saeid Sedighi, Bhavani Shankar Mysore R, Sina Maleki, and Björn Ottersten \\ Interdisciplinary Centre for Security, Reliability and Trust (SnT), University of Luxembourg \\ E-mails: \{saeid.sedighi,bhavani.shankar,sina.maleki,bjorn.ottersten\}@uni.lu
}

\begin{abstract}
Sparse linear arrays (SLAs), such as nested and co-prime arrays, have the attractive capability of providing enhanced degrees of freedom by exploiting the co-array model. Accordingly, co-array-based Direction of Arrivals (DoAs) estimation has recently gained considerable interest in array processing. The literature has suggested applying MUSIC on an augmented sample covariance matrix for co-array-based DoAs estimation. In this paper, we propose a Least Squares (LS) estimator for co-array-based DoAs estimation employing the covariance fitting method as an alternative to MUSIC. We show that the proposed LS estimator provides consistent estimates of DoAs of identifiable sources for SLAs. Additionally, an analytical expression for the large sample performance of the proposed estimator is derived. Numerical results illustrate the finite sample behavior in relation to the derived analytical expression. Moreover, the performance of the proposed LS estimator is compared to the co-array-based MUSIC.
\end{abstract}

Index Terms - Sparse linear arrays, directions of arrival estimation, least squares estimator, performance analysis, consistency.

\section{INTRODUCTION}

The problem of Directions of Arrivals (DoAs) estimation is of central importance in the field of array processing with many applications in radar, sonar, and wireless communications [1-3]. DoAs estimation using Uniform Linear Arrays (ULAs) is well investigated in the literature. In this regard, a variety of algorithms such as Maximum Likelihood (ML) estimation, MUSIC, ESPRIT and subspace fitting has been presented and thoroughly analyzed in [4-7]. It is known that a ULA with $M$ elements can identify up to $M-1$ uncorrelated sources [2,6]. However, exploiting Sparse Linear Arrays (SLAs) such as Minimum Redundancy Arrays (MRAs) [8] can dramatically boost the degrees of freedom such that significantly more sources than the number of elements in the array can be identified. Despite this feature, SLAs received little attention for a long time due to complexity in finding MRAs geometries.

Recently, the introduction of new SLA architectures with simple closed-from expression for the array geometry, such as co-prime arrays [9] and nested arrays [10], has spurred a renewed interest in SLAs. These arrays allow for identification of up to $\mathcal{O}\left(M^{2}\right)$ uncorrelated sources with only $M$ elements in the array by exploiting the difference co-array model $[9,10]$. In a very recent paper, the authors in [11] have provided new insights into the performance limits of co-array-based DoAs estimation through an analysis of the Cramér-Rao Bound (CRB). The co-array model consists of a single

This work is supported by the National Research Fund, Luxembourg under AFR grant for bilateral Ph.D. project (Project Reference 11228830) on "Compressive Sensing for Ranging and Detection in Automotive Applications". snapshot requiring preprocessing steps before an application of conventional DoAs estimation methods. For instance, spatial smoothing combined with the MUSIC algorithm has been proposed in [10]. However, since the augmented covariance matrix constructed after spatial smoothing has statistical properties different from the original sample covariance matrix, the performance analysis of conventional MUSIC cannot be applied directly to co-array-based MUSIC. Motivated by this fact, the authors in [12] recently presented an asymptotic mean-squared error (MSE) expression for the co-array-based MUSIC algorithm and investigated its behavior in various scenarios.

In this paper, we propose a Least Squared (LS) estimator for coarray-based DoAs estimation using the covariance fitting method. We first demonstrate consistency of the proposed LS estimator. Building on consistency of the proposed LS estimator, we then derive an asymptotic closed-form expression for its covariance matrix. This provides us with insights into the impact of different system parameters on the performance of the proposed LS estimator. Further, we validate the analytical derivations through numerical simulations besides comparing the performance of the proposed LS estimator with co-array-based MUSIC. The simulations reveal that the LS estimator largely has a performance comparable to coarray-based MUSIC while slightly outperforming the latter in some scenarios.

Notation: Vectors and matrices are referred to by lower- and upper-case bold-face, respectively. The superscripts $*, T, H$ denote the conjugate, transpose and Hermitian (conjugate transpose) operations, respectively. $\|\mathbf{A}\|_{F}$ and $\|\mathbf{a}\|_{2}$ stand for the Frobeniusand $\ell_{2}$-norm of $\mathbf{A}$ and $\mathbf{a}$, respectively. $[\mathbf{A}]_{i, j}$ and $[\mathbf{a}]_{i}$ indicate the $(i, j)^{\text {th }}$ and $i^{\text {th }}$ entry of $\mathbf{A}$ and $\mathbf{a}$, respectively. $\hat{\mathbf{A}}$ and $\hat{\mathbf{a}}$ denote the estimate of $\mathbf{A}$ and $\mathbf{a}$, respectively. $\left(a_{1}, a_{2}, \cdots, a_{n}\right)$ is an $n$ tuple with elements of $a_{1}, a_{2}, \cdots, a_{n} .|\mathbb{A}|$ represents the cardinality of the set $\mathbb{A}$. $\operatorname{diag}(\mathbf{a})$ is a diagonal matrix whose diagonal entries are equal to those of a. The $M \times M$ identity matrix is denoted by $\mathbf{I}_{M} . \mathbb{E}\{$.$\} stands for the statistical expectation. \otimes$ and $\odot$ represent Kronecker and Khatri-Rao products, respectively. $\operatorname{Vec}(\mathbf{A})=\left[\begin{array}{llll}\mathbf{a}_{1}^{T} & \mathbf{a}_{2}^{T} & \cdots & \mathbf{a}_{n}^{T}\end{array}\right]^{T}$ denotes the vectorization operation. $\mathbf{A}^{\dagger}$ and $\Pi_{\mathbf{A}}^{\perp}$ indicate the pseudoinverse and the projection matrix onto the null space of the full column rank matrix $\mathbf{A}$, respectively.

\section{CO-ARRAY SYSTEM MODEL}

We consider an SLA with $M$ elements located at positions $\left(m_{1} \frac{\lambda}{2}\right.$, $\left.m_{2} \frac{\lambda}{2}, \cdots, m_{M} \frac{\lambda}{2}\right)$ with $m_{i} \in \mathbb{M}$. Here $\mathbb{M}$ is an integer set with cardinality $|\mathrm{M}|=M$, and $\lambda$ represents the wavelength of the incoming signals. It is assumed $K$ narrowband signals with distinct DOAs $\boldsymbol{\theta}=\left[\begin{array}{llll}\theta_{1} & \theta_{2} & \cdots & \theta_{K}\end{array}\right]^{T}$ impinge on the SLA from far field. Accordingly, the vector of signals received by the SLA at time 
instance $t$ can be modeled as

$$
\mathbf{y}(t)=\mathbf{A}(\boldsymbol{\theta}) \mathbf{x}(t)+\mathbf{n}(t) \in \mathbb{C}^{M \times 1}, \quad t=1, \cdots, N,
$$

where $\mathbf{x}(t) \in \mathbb{C}^{K \times 1}$ denotes the signal vector, $\mathbf{n}(t) \in \mathbb{C}^{M \times 1}$ is additive noise, and $\mathbf{A}(\boldsymbol{\theta})=\left[\begin{array}{llll}\mathbf{a}\left(\theta_{1}\right), & \mathbf{a}\left(\theta_{2}\right), & \cdots & \mathbf{a}\left(\theta_{K}\right)\end{array}\right] \in$ $\mathbb{C}^{M \times K}$ represents the SLA manifold matrix where

$$
\mathbf{a}\left(\theta_{i}\right)=\left[\begin{array}{llll}
e^{j \pi \theta_{i} m_{1}}, & e^{j \pi \theta_{i} m_{2}}, & \cdots & e^{j \pi \theta_{i} m_{M}}
\end{array}\right]^{T},
$$

is the SLA steering vector for the $k^{\text {th }}$ signal and $\theta_{k}=\sin \tilde{\theta}_{k}$ its normalized DoA. The following assumptions are made on the source signals and noise:

A1 The noise vector is assumed zero-mean circular complex Gaussian distributed with the covariance matrix $\mathbb{E}\left\{\mathbf{n}(t) \mathbf{n}^{H}(t)\right\}=$ $\sigma^{2} \mathbf{I}_{M}$.

A2 The signal vector is modeled as a zero-mean circular complex Gaussian random vector with covariance matrix $\mathbb{E}\left\{\mathbf{x}(t) \mathbf{x}^{H}(t)\right\}$ $=\operatorname{diag}\{\mathbf{p}\}$ where $\mathbf{p}=\left[\begin{array}{llll}p_{1}, & p_{2}, & \cdots & p_{K}\end{array}\right]^{T} \in \mathbb{R}^{+^{K \times 1}}$.

A3 The signal and noise vectors are assumed mutually independent.

A4 There is no temporal correlation between the snapshots.

Based on the above assumptions, the covariance matrix of the received signals, $\mathbf{R}=\mathbb{E}\left\{\mathbf{y}(t) \mathbf{y}^{H}(t)\right\}$, is given by

$$
\mathbf{R}=\mathbf{A}(\boldsymbol{\theta}) \operatorname{diag}(\mathbf{p}) \mathbf{A}^{H}(\boldsymbol{\theta})+\sigma^{2} \mathbf{I}_{M} \in \mathbb{C}^{M \times M} .
$$

Following [10-12], the difference co-array model of the SLA is obtained by using the following standard steps. At first, $\mathbf{r}=$ $\operatorname{Vec}(\mathbf{R}) \in \mathbb{C}^{M^{2} \times 1}$, the vectorized form of $\mathbf{R}$ takes the form

$$
\mathbf{r}=\left(\mathbf{A}^{*}(\boldsymbol{\theta}) \odot \mathbf{A}(\boldsymbol{\theta})\right) \mathbf{p}+\sigma^{2} \operatorname{Vec}(\mathbf{I}) .
$$

Subsequently, duplicated entries of $\mathbf{r}$ are eliminated to obtain the difference co-array model as

$$
\mathbf{z}=\mathbf{J r}=\mathbf{A}_{d}(\boldsymbol{\theta}) \mathbf{p}+\sigma^{2} \mathbf{g},
$$

where $\mathbf{A}_{d}(\boldsymbol{\theta}) \in \mathbb{C}^{D \times K}$ corresponds to the array manifold matrix of the virtual array whose elements are located at $\left(\ell_{1} \frac{\lambda}{2}, \ell_{2} \frac{\lambda}{2}, \cdots, \ell_{D} \frac{\lambda}{2}\right)$ with $\ell_{i} \in \mathbb{D}=\left\{m_{p}-m_{q} \mid m_{p}, m_{q} \in \mathbb{M}\right\}, D=|\mathbb{D}|$, $\mathbf{g}$ is a column vector defined as $[\mathbf{g}]_{i}=\delta[i]$, and $\mathbf{J}$ represents a $\{0,1\}^{D \times M^{2}}$ selection matrix defined as

$$
[\mathbf{J}]_{n, p+(q-1) M}=\left\{\begin{array}{cc}
\frac{1}{w\left(\ell_{n}\right)}, & \text { if } m_{p}-m_{q}=\ell_{n}, \\
0, & \text { otherwise, }
\end{array} .\right.
$$

with $w\left(\ell_{n}\right)=\left|\mathbb{A}\left(\ell_{n}\right)\right|$ where $\mathbb{A}\left(\ell_{n}\right)=\left\{\left(m_{p}, m_{q}\right) \in \mathbb{M}^{2} \mid m_{p}-\right.$ $\left.m_{q}=\ell_{n}\right\}$. The difference co-array model in (5) can be reckoned to be the response of the virtual array described by $\mathbf{A}_{d}(\boldsymbol{\theta})$ to the deterministic signals vector $\mathbf{p}$. If the SLA is designed properly, $\mathbf{A}_{d}(\boldsymbol{\theta})$ contains a contiguous ULA segment around the origin whose number of elements, i.e., $\vartheta$, is much larger than the number of physical elements in the SLA, enabling us to identify more sources than the number of physical elements in the SLA $[9,10]$.

To estimate source DoAs based on the co-array model in (5), it is possible to form an augmented covariance matrix and then apply MUSIC to it $[10,12]$. One possible way to obtain the augmented covariance matrix is to divide the contiguous ULA segment of the virtual array, described by its manifold matrix $\mathbf{A}_{\vartheta}(\boldsymbol{\theta}) \in \mathbb{C}^{\vartheta \times K}$, into $v=\frac{\vartheta+1}{2}$ overlapping subarrays, each with $v$ elements, such that the elements of the $i^{\text {th }}$ subarray are situated at positions $\left((-i+1) \frac{\lambda}{2}, \cdots,(v-i) \frac{\lambda}{2}\right)$ where $1 \leq i \leq v$. The output of the $i^{\text {th }}$ subarray can be expressed as $\mathbf{z}_{i}=\Gamma_{i} \mathbf{z}=\boldsymbol{\Gamma}_{i} \mathbf{J r}$, where $\boldsymbol{\Gamma}_{i}=\left[\begin{array}{lll}\mathbf{0}_{v \times\left(\frac{D+1}{2}-i\right)} & \mathbf{I}_{v} & \mathbf{0}_{v \times\left(i+\frac{D-\vartheta}{2}-1\right)}\end{array}\right] \in\{0,1\}^{v \times D}$. Then, the augmented covariance matrix of the co-array model, defined as $\mathbf{R}_{v}=\left[\begin{array}{llll}\mathbf{z}_{v} & \mathbf{z}_{v-1} & \cdots & \mathbf{z}_{1}\end{array}\right]$, can be formed as follows [13]

$$
\mathbf{R}_{v}=\mathbf{A}_{v}(\boldsymbol{\theta}) \operatorname{diag}(\mathbf{p}) \mathbf{A}_{v}^{H}(\boldsymbol{\theta})+\sigma^{2} \mathbf{I},
$$

where $\mathbf{A}_{v}(\boldsymbol{\theta}) \in \mathbb{C}^{v \times K}$ is the array manifold matrix of the first subarray. The matrix $\mathbf{R}_{v}$ has the same structure as the covariance matrix of the signal received by a ULA with $v$ elements, hence by applying MUSIC to it up to $v-1$ source DoAs can be estimated.

However, in practice, the true value of $\mathbf{R}$, and consequently, $\mathbf{r}, \mathbf{z}$, and $\mathbf{R}_{v}$ are not available. Therefore, we need to replace them with their consistent estimates. $\mathbf{R}$ can be consistently estimated by the sample covariance matrix $\hat{\mathbf{R}}=\frac{1}{N} \sum_{t=1}^{N} \mathbf{y}(t) \mathbf{y}^{H}(t)$. Consequently, we can also find the consistent estimates of $\mathbf{r}, \mathbf{z}, \mathbf{R}_{v}$ by substituting $\hat{\mathbf{R}}$ for $\mathbf{R}$ in (4), (5), and (7).

\section{CO-ARRAY-BASED LS ESTIMATOR AND ITS PERFORMANCE}

\subsection{Formulation of LS Estimator in Co-Array Scenario}

In this section, as an alternative to co-array-based MUSIC, we derive the LS estimates of source DOAs based on the co-array model and analyze its performance. Using the augmented covariance matrix introduced in Section 2, we can formulate the co-array-based LS estimates of $\boldsymbol{\theta}, \mathbf{p}, \sigma^{2}$ as

$\left[\widehat{\boldsymbol{\theta}}_{l s}^{T}, \widehat{\mathbf{p}}_{l s}^{T}, \widehat{\sigma}_{l s}^{2}\right]^{T}=\underset{\boldsymbol{\theta}, \mathbf{p}, \sigma^{2}}{\operatorname{argmin}}\left\|\hat{\mathbf{R}}_{v}-\mathbf{A}_{v}(\boldsymbol{\theta}) \operatorname{diag}(\mathbf{p}) \mathbf{A}_{v}^{H}(\boldsymbol{\theta})-\sigma^{2} \mathbf{I}\right\|_{F}^{2}$.

Using the separability property [14], we can first solve (8) with respect to $\sigma^{2}$ and then substitute the result into (8). To this end, we rewrite (8) as

$$
\left[\widehat{\boldsymbol{\theta}}_{l s}^{T}, \widehat{\mathbf{p}}_{l s}^{T}, \widehat{\sigma}_{l s}^{2}\right]^{T}=\underset{\boldsymbol{\theta}, \mathbf{p}, \sigma^{2}}{\operatorname{argmin}}\left\|\hat{\mathbf{R}}_{v}-\mathbf{U}_{s} \boldsymbol{\Lambda} \mathbf{U}_{s}^{H}-\sigma^{2} \mathbf{U}_{n} \mathbf{U}_{n}^{H}\right\|_{F}^{2},
$$

where $\mathbf{U}_{s}, \mathbf{U}_{n}$ are the eigenvectors of $\mathbf{R}_{v}$ corresponding to its $K$ largest and $v-K$ smallest eigenvalues, respectively. Equation (9) leads to the following estimate of $\sigma^{2}$

$$
\widehat{\sigma}_{l s}^{2}=\frac{\operatorname{tr}\left\{\hat{\mathbf{U}}_{n} \hat{\mathbf{U}}_{n}^{H} \hat{\mathbf{R}}_{v}\right\}}{\operatorname{tr}\left\{\hat{\mathbf{U}}_{n} \hat{\mathbf{U}}_{n}^{H}\right\}}=\frac{\operatorname{tr}\left\{\hat{\mathbf{U}}_{n} \hat{\mathbf{U}}_{n}^{H} \hat{\mathbf{R}}_{v}\right\}}{v-K},
$$

where $\hat{\mathbf{U}}_{s}$ represents the eigenvectors of $\hat{\mathbf{R}}_{v}$ corresponding to its $v-K$ smallest eigenvalues.

Remark 1. Using consistency of $\hat{\mathbf{R}}_{v}$, it can be readily deduced that $\hat{\sigma}^{2}$ is a consistent estimate of $\sigma^{2}$ [14].

Inserting (10) into (8) and performing certain algebraic manipulations leads to

$$
\left[\widehat{\boldsymbol{\theta}}_{l s}^{T}, \widehat{\mathbf{p}}_{l s}^{T}\right]^{T}=\underset{\boldsymbol{\theta}, \mathbf{p}}{\operatorname{argmin}}\left\|\hat{\mathbf{Q}} \operatorname{Vec}\left(\hat{\mathbf{R}}_{v}\right)-\left(\mathbf{A}_{v}^{*}(\boldsymbol{\theta}) \odot \mathbf{A}_{v}(\boldsymbol{\theta})\right) \mathbf{p}\right\|_{2}^{2},
$$

where $\hat{\mathbf{Q}}=\mathbf{I}-\frac{\operatorname{Vec}(\mathbf{I}) \operatorname{Vec}^{H}\left(\hat{\mathbf{U}}_{n} \hat{\mathbf{U}}_{n}^{H}\right)}{v-K}$. Towards further simplifying (11) to obtain $\widehat{\mathbf{p}}_{l s}$, it can be shown from (2) and (7) that

$$
\operatorname{Vec}\left(\hat{\mathbf{R}}_{v}\right)=\operatorname{Vec}\left(\left[\begin{array}{llll}
\boldsymbol{\Gamma}_{v} \mathbf{z} & \boldsymbol{\Gamma}_{v-1} \mathbf{z} & \cdots & \boldsymbol{\Gamma}_{1} \mathbf{z}
\end{array}\right]\right)=\boldsymbol{\Gamma} \mathbf{J} \hat{\mathbf{r}},
$$


where $\boldsymbol{\Gamma}=\left[\begin{array}{lll}\boldsymbol{\Gamma}_{v}^{T} & \boldsymbol{\Gamma}_{v-1}^{T} & \cdots\end{array} \boldsymbol{\Gamma}_{1}^{T}\right]^{T}$. In addition, it can be shown that

$$
\mathbf{A}_{v}^{*}(\boldsymbol{\theta}) \odot \mathbf{A}_{v}(\boldsymbol{\theta})=\mathbf{T} \mathbf{A}_{\vartheta}(\boldsymbol{\theta}),
$$

where $\mathbf{T} \in\{0,1\}^{v^{2} \times \vartheta}$ is the selection matrix, defined as

$$
[\mathbf{T}]_{p+(q-1) v, n}=\left\{\begin{array}{lc}
1, & \text { if } p-q=n-v, \\
0, & \text { otherwise, }
\end{array} .\right.
$$

with $1 \leq p, q \leq v$. Inserting (12) and (13) into (11), we get

$$
\left[\widehat{\boldsymbol{\theta}}_{l s}^{T}, \widehat{\mathbf{p}}_{l s}^{T}\right]^{T}=\underset{\boldsymbol{\theta}, \mathbf{p}}{\operatorname{argmin}}\left\|\hat{\mathbf{Q}} \boldsymbol{\Gamma} \mathbf{J} \hat{\mathbf{r}}-\mathbf{T} \mathbf{A}_{\vartheta}(\boldsymbol{\theta}) \mathbf{p}\right\|_{2}^{2} .
$$

Solving (15) with respect to $\mathbf{p}$ yields

$$
\widehat{\mathbf{p}}_{l s}=\left(\mathbf{T A}_{\vartheta}(\boldsymbol{\theta})\right)^{\dagger} \hat{\mathbf{Q}} \boldsymbol{\Gamma} \mathbf{J} \hat{\mathbf{r}} .
$$

Remark 2. Similar to $\widehat{\sigma}_{l s}^{2}$, consistency of $\widehat{\mathbf{p}}_{l s}$ can also be deduced from consistency of $\hat{\mathbf{r}}$ and $\hat{\mathbf{R}}_{v}$ [14].

Finally, inserting (16) into (15), results in the LS estimator of $\boldsymbol{\theta}$,

$\hat{\boldsymbol{\theta}}_{l s}=\underset{\boldsymbol{\theta}}{\operatorname{argmin}}\left\|\Pi_{\mathbf{T} \mathbf{A}_{\vartheta}(\boldsymbol{\theta})}^{\perp}\left(\mathbf{I}-\frac{\operatorname{Vec}(\mathbf{I}) \operatorname{Vec}^{H}\left(\hat{\mathbf{U}}_{n} \hat{\mathbf{U}}_{n}^{H}\right)}{v-K}\right) \boldsymbol{\Gamma} \mathbf{J} \hat{\mathbf{r}}\right\|_{2}^{2}$.

In general, the above optimization is a nonlinear problem, which seems to be computationally complex. However, it is possible to be reparameterized as a linear problem in a manner similar to [15], where the DoA estimates are obtained by finding roots of a polynomial function.

Having derived the estimator, we enumerate the properties influencing its performance.

\subsection{Properties of LS Estimator}

In the following, we comment on the key attributes of the LS estimator including its consistency, bias and covariance matrix. Asymptotics are resorted to yield tractable analytical results.

Lemma 1. $\hat{\boldsymbol{\theta}}_{l s}$ is a consistent estimator of $\boldsymbol{\theta}$ if $K \leq v-1$.

Proof. The proof is omitted due to lack of space.

Remark 3. Unbiased It readily follows from Lemma 1 that $\hat{\boldsymbol{\theta}}_{l s}$ is asymptotically unbiased.

Remark 4. Relation to the CRB The condition $K \leq v-1$ in Lemma 1 corresponds to the rank condition mentioned in [11] for the nonsigularity of Fisher information matrix. Hence, it can be concluded that the LS estimator is consistent whenever the CRB exists.

We exploit consistency of $\widehat{\boldsymbol{\theta}}_{l s}$ to obtain its asymptotic covariance matrix, which is given in the following theorem.

Theorem 1. If $K \leq v-1$, the asymptotic $(N \rightarrow \infty)$ covariance matrix of the LS estimator $\widehat{\boldsymbol{\theta}}_{l s}$ is given by

$$
\begin{aligned}
& \mathbf{C}_{l s}=\mathbb{E}\left\{\left(\hat{\boldsymbol{\theta}}_{l s}-\boldsymbol{\theta}\right)\left(\hat{\boldsymbol{\theta}}_{l s}-\boldsymbol{\theta}\right)^{H}\right\}=\frac{1}{\pi^{2} N} \times \\
& \left(\operatorname{diag}(\mathbf{p}) \boldsymbol{\Omega}^{H} \Pi_{\mathbf{T} \mathbf{A}_{\vartheta}(\boldsymbol{\theta})}^{\perp} \boldsymbol{\Omega}\right. \\
& \times\left(\boldsymbol{\Omega}^{H} \Pi_{\mathbf{T} \mathbf{A}_{\vartheta}(\boldsymbol{\theta})}^{\perp} \mathbf{Q} \boldsymbol{\Gamma} \mathbf{J}\left(\mathbf{R}^{T} \otimes \mathbf{R}\right) \mathbf{J}^{H} \boldsymbol{\Gamma}^{H} \mathbf{Q}^{H} \Pi_{\mathbf{T} \mathbf{A}_{\vartheta}(\boldsymbol{\theta})}^{\perp} \boldsymbol{\Omega}\right)^{-1} \\
& \left.\times \boldsymbol{\Omega}_{\mathbf{T} \mathbf{A}_{\vartheta}(\boldsymbol{\theta})}^{\perp} \boldsymbol{\Omega}^{H} \operatorname{diag}(\mathbf{p})\right)^{-1}
\end{aligned}
$$

where $\boldsymbol{\Omega}=\mathbf{T} \operatorname{diag}(\boldsymbol{\vartheta}) \mathbf{A}_{\vartheta}(\boldsymbol{\theta})$ with $\boldsymbol{\vartheta}=[0,1,2, \cdots, \vartheta-1]^{T}$.
Proof. The proof is omitted due to the lack of space.

Remark 5. Dependence on array geometry Theorem 1 implies that the MSE of the LS estimator is dependent on both of the physical and virtual arrays geometries through $\mathbf{A}_{\vartheta}(\boldsymbol{\theta})$ and $\mathbf{J}\left(\mathbf{R} \otimes \mathbf{R}^{T}\right) \mathbf{J}^{H}$, respectively. This means that two SLAs with identical virtual array geometries could still have different MSEs.

In addition, it can be deduced from Theorem 1 that the MSE of the LS estimator depends on the Signal-to-Noise Ratios (SNRs) instead of source powers and noise variance. In order to assess the impact of the SNR on the MSE of the proposed estimator, we introduce the following corollary.

Corollary 1. Assuming the powers of all sources are equal, each entry of $\mathbf{C}_{l s}$ decreases monotonically as SNR increases, and has the following asymptotic behavior,

1. $\lim _{S N R \rightarrow \infty} \mathbf{C}_{l s}=0$ if $K=1$.

2. $\lim _{S N R \rightarrow \infty} \mathbf{C}_{l s} \succeq 0$ if $2 \leq K<M$.

3. $\lim _{S N R \rightarrow \infty} \mathbf{C}_{l s} \succeq 0$ if $K \geq M$ and $\operatorname{rank}(\mathbf{A}(\boldsymbol{\theta}))<M$.

4. $\lim _{S N R \rightarrow \infty} \mathbf{C}_{l s} \succ 0$ if $K \geq M$ and $\operatorname{rank}(\mathbf{A}(\boldsymbol{\theta}))=M$.

Corollary 1 indicates that the asymptotic behavior of the LS estimator with respect to the SNR affected by the actual value of source DoAs when multiple sources are present. Indeed, for some DoA values, $\mathbf{C}_{l s}$ could be saturated and could monotonically decrease for others.

\section{SIMULATION RESULTS}

In this section, we numerically analyze the performance of the proposed LS estimator. Throughout this section, we consider nested arrays with $M=M_{1}+M_{2}$ elements whose locations are given by

$\mathbb{M}=\left\{1,2 \cdots, M_{1}\right\} \cup\left\{M_{1}+1,2\left(M_{1}+1\right) \cdots, M_{2}\left(M_{1}+1\right)\right\}$.

The sources are also assumed to have equal powers and normalized DoAs evenly selected between -0.4 and 0.4 . In addition, in all experiments, 1000 independent Monte Carlo realizations are used to generate the empirical plots.

Fig. 1 depicts the MSE versus the number of snapshots at SNR $=5 \mathrm{~dB}$ when $K=7$ sources impinge on a nested array with $\frac{M}{2}=M_{1}=M_{2}=3$. It can be seen that the LS estimator performs slightly better than co-array-based MUSIC in this case, but the MSEs of both are far from the CRB indicating their statistical inefficiency. Moreover, Fig. 1 reveals that there is a good agreement between the analytical MSE obtained using Theorem 1 and the empirical results even for the finite number of snapshots.

Fig 2 shows the MSE of the LS estimator versus the SNR for nested arrays with different physical array but the same virtual array geometries. We set $N=500$. The number of sources, i.e., $K$, is selected so as to be respectively smaller and greater than the number of physical array elements in Figs. 2(a) and 2(b). It can be seen that while the nested arrays share the virtual array geometry, they have different MSEs due to difference in their physical array geometries. These observations agree with Remark 5. In addition, Fig. 2 shows the relationship between the MSE and the SNR. It can be observed that for both of the cases investigated in Figs. 2(a) and 2(b) , i.e., $K=3$ and $K=13$, the MSE monotonically decreases with increase in the SNR, but is eventually saturated at some point. This behavior was already predictable considering Corollary 1 . 


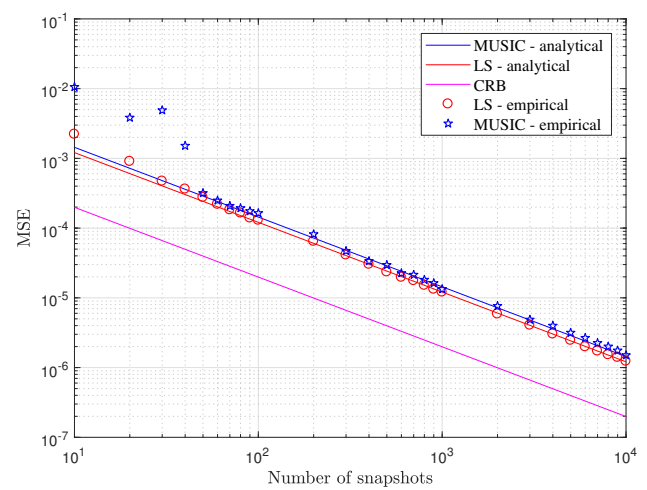

Fig. 1. MSE versus the number of snapshots for $\mathrm{SNR}=5 \mathrm{~dB}$, $K=7$, and a nested array with $\frac{M}{2}=M_{1}=M_{2}=3$.

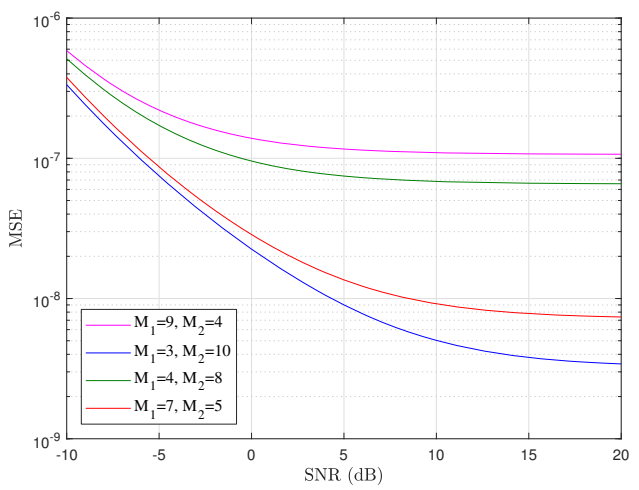

(a)

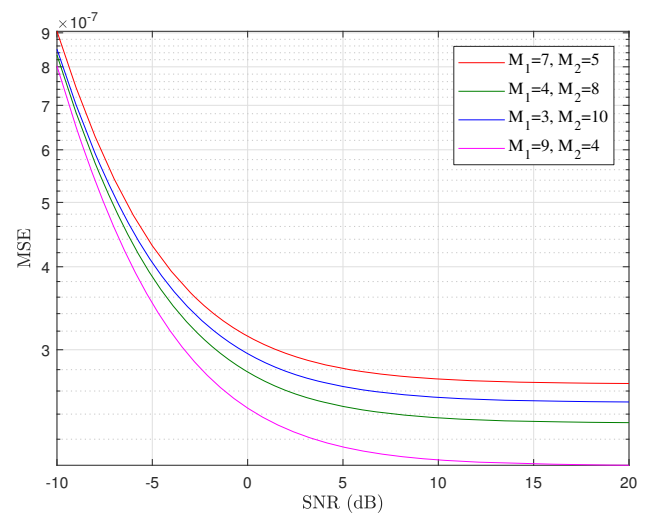

(b)

Fig. 2. MSE versus the SNR for different nested array configurations with identical virtual array geometries. The number of snapshots is $N=500$, and: (a) $M>K=3$ (b) $M \leq K=13$.

In Fig 3, we plot the MSE versus the SNR for 500 snapshots. We consider $K=5$ sources whose DoAs are estimated by a nested array with $\frac{M}{2}=M_{1}=M_{2}=5$. It is seen that, as opposed to the MSEs of the LS estimator and co-array-based MUSIC saturated when the SNR is over $10 \mathrm{~dB}$, the CRB goes to zero with increase in the SNR. This behavior of the CRB with respect to the SNR for $K<M$ was already predicted in [11, Theorems 3]. This implies that both of the LS estimator and co-array-based MUSIC can become highly

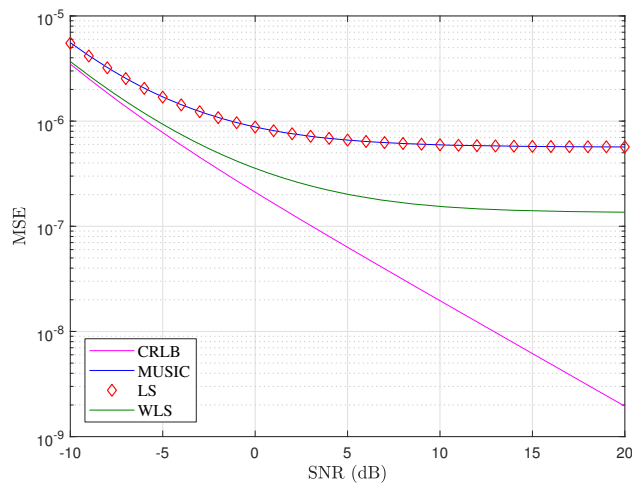

Fig. 3. MSE versus the SNR for $N=500$, and $K=5$, and a nested array with $\frac{M}{2}=M_{1}=M_{2}=5$.

statistical inefficient in the high SNR regime. Fig 3 also shows the MSE of a Weighted LS (WLS) estimator, heuristically defined as

$$
\hat{\boldsymbol{\theta}}_{w l s}=\underset{\boldsymbol{\theta}}{\operatorname{argmin}}\left\|\mathbf{W}^{\frac{1}{2}} \Pi_{\mathbf{T} \mathbf{A}_{\vartheta}(\boldsymbol{\theta})}^{\perp} \hat{\mathbf{Q}} \boldsymbol{\Gamma} \mathbf{J} \hat{\mathbf{r}}\right\|_{2}^{2},
$$

with $\mathbf{W}$ being a positive definite Hermitian matrix. We see that WLS estimator is superior to both of the LS estimator and co-array-based MUSIC. This is just a preliminary investigation of the prospect of performance improvement over the LS estimator and co-array-based MUSIC using a weighting matrix. However, determination of the optimal weighting matrix remains as the topic of the future work.

\section{CONCLUSION}

In this paper, we proposed a Least Squares (LS) estimator for co-array-based DoAs estimation employing the covariance fitting method and analyzed it performance. It was shown that the proposed LS estimator provides consistent estimates of DoAs of identifiable sources for SLAs. Further, the relationship between the MSE of the proposed LS estimator and the SNR was investigated by exploiting the analytical expression derived for the large sample performance of the proposed estimator. Numerical results showed that the LS estimator has a comparable performance to co-array-based MUSIC, but preliminary investigation of a weighted LS variant with heuristic weights was undertaken and the results were better than the LS estimator and MUSIC. The optimization of these weights is left for the future work.

\section{REFERENCES}

[1] H. Van Trees, Optimum Array Processing (Detection, Estimation, and Modulation Theory, Part IV). New York: John Wiley and Sons Inc., 2002.

[2] S. S. Haykin, J. Litva, , and T. J. Shepherd, Eds., Radar Array Processing. Berlin, Germany: Springer-Verlag, 1993.

[3] B. Ottersten, "Array processing for wireless communications," in Proceedings of 8th Workshop on Statistical Signal and Array Processing, Jun 1996, pp. 466-473.

[4] A. Paulraj, B. Ottersten, R. Roy, A. Swindlehurst, G. Xu, and T. Kailath, "16 subspace methods for directions-of-arrival estimation,” Handbook of Statistics, vol. 10, pp. 693-739, 1993. 
[5] F. Li, H. Liu, and R. J. Vaccaro, "Performance analysis for doa estimation algorithms: unification, simplification, and observations," IEEE Transactions on Aerospace and Electronic Systems, vol. 29, no. 4, pp. 1170-1184, Oct 1993.

[6] P. Stoica and A. Nehorai, "Performance study of conditional and unconditional direction-of-arrival estimation," IEEE Transactions on Acoustics, Speech, and Signal Processing, vol. 38, no. 10, pp. 1783-1795, Oct 1990.

[7] M. Viberg, B. Ottersten, and T. Kailath, "Detection and estimation in sensor arrays using weighted subspace fitting," IEEE Transactions on Signal Processing, vol. 39, no. 11, pp. 24362449, Nov 1991.

[8] A. Moffet, "Minimum-redundancy linear arrays," IEEE Transactions on Antennas and Propagation, vol. 16, no. 2, pp. 172175, Mar 1968.

[9] P. P. Vaidyanathan and P. Pal, "Sparse sensing with co-prime samplers and arrays," IEEE Transactions on Signal Processing, vol. 59, no. 2, pp. 573-586, Feb 2011.

[10] P. Pal and P. P. Vaidyanathan, "Nested arrays: A novel approach to array processing with enhanced degrees of freedom," IEEE Transactions on Signal Processing, vol. 58, no. 8, pp. 4167-4181, Aug 2010.

[11] C. L. Liu and P. Vaidyanathan, "Cramér-rao bounds for coprime and other sparse arrays, which find more sources than sensors," Digital Signal Processing, vol. 61, pp. 43 - 61, 2017.

[12] M. Wang and A. Nehorai, "Coarrays, music, and the cram érrao bound," IEEE Transactions on Signal Processing, vol. 65, no. 4, pp. 933-946, Feb 2017.

[13] C. L. Liu and P. P. Vaidyanathan, "Remarks on the spatial smoothing step in coarray music," IEEE Signal Processing Letters, vol. 22, no. 9, pp. 1438-1442, Sept 2015.

[14] S. M. Kay, Fundamentals of statistical signal processing, Volume I: Estimation theory. Englewood Cliffs, NJ: Prentice Hall, 1993.

[15] P. Stoica and K. C. Sharman, "Maximum likelihood methods for direction-of-arrival estimation," IEEE Transactions on Acoustics, Speech, and Signal Processing, vol. 38, no. 7, pp. 1132-1143, Jul 1990. 